\title{
ANALYSIS OF 11 LATE-TYPE CLOSE BINARY SYSTEMS*
}

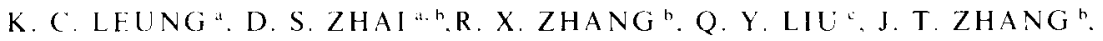 \\ and Y. L. YANG
}

(Received 18 July, 1983)

\begin{abstract}
This is a preliminary report on the joint research project between 3 observatories: Beijing, Yunnan, and Behlen Observatories from China and the United States. The systems we have been dealing with are primary of late spectral types and with short periods. Most.of the observations were secured from the observatories in China. The computational analysis is carried out in University of Nebraska, Lincoln. The photometric solutions are based on the Wilson and Devinney method. Out of 11 systems analyzed 6 of them: AO Cam. ER Ori, BX Peg, BB Peg, U Peg, and SW Lac are found to be contact systems. All of them are having their primary eclipses at occultation. Therefore they can be classified as W-type W UMa systems. They also show other W-type characteristics.

Three systems: ZZAur, RZ Dra, and AXVir are found to be semi-detached systems, with low mass components filling their Roche surfaces. The massive components are having their radii fairly close to Roche surfaces and are larger than their contact companions.

The last two systems: AT Cam and AZCam are found to be detached systems, but with their low mass components almost filling their Roche surfaces. This type of configuration is of great interest to our understanding of close binary evolution. We believe that these systems are at phase immediately after the normal Algol phase, where the mass lossing components detached from the Roche surfaces at the conclusion active mass lost phase.
\end{abstract}

\section{Acknowledgements}

We would like to acknowledge the support of the NSF INT 8120404 grant, and the Academia Sinica.

\footnotetext{
* Paper presented at the Lembang-Bamberg IAU Colloquium No. 80 on 'Double Stars: Physical Properties and Generic Relations', held at Bandung, Indonesia, 3- 7 June, 1983.

Behlen Observatory, University of Nebraska, Lincoln, Nebr., U.S.A.

b Beijing Observatory, Academia Sinica, Beijing, P.R.C.

c Yunnan Observatory, Academia Sinica, Kunming, P.R.C.
} 